\title{
Acoustic pressure contributions of a combine harvester's thresher drum to the overall workplace noise situation
}

\author{
Valentin Stepanov ${ }^{1^{*}}$, Sergey Kireev $^{2}$, and Marina Korchagina $^{2}$ \\ ${ }^{1}$ JSC “Rostov Civil Aircraft Factory №412”, 282, Sholokhov Ave., Rostov-on-Don, 344099, Russia \\ Federation \\ ${ }^{2}$ Don State Technical University, 1. Gagarina Square, Rostov-on-Don, 344000, Russian Federation
}

\begin{abstract}
This paper analyzes the magnitude of the acoustic pressure contribution of a combine harvester's thresher drum to the overall noise situation with the goal of providing the regulatory parameters for the workplace conditions. In the course of the current research the velocity and pressure graphics for the airflow on the parts of a rotating thresher drum as well as the sound intensity parameters by frequency spectra and sound pressure magnitudes were calculated.
\end{abstract}

\section{Introduction}

The relevance of the problem of noise reduction is associated with the normalized regulation of the permissible sound level at the workplace of $80 \mathrm{~dB}$ (A) in Russia, whereas in the EU the maximum permissible level is $87 \mathrm{~dB}(\mathrm{~A})$, and the upper threshold level is 85 $\mathrm{dB}(\mathrm{A})$.

A human reaction to sound as an irritant is due to the physiological characteristics of human hearing organs. The sound pressure is perceived as the difference between the instant value of pressure and its mean value. The change in sound intensity is defined as the logarithm of the amount of energy transferred. The frequency spectrum is defined as the range of audible sound in octave bands. This imposes certain requirements on the methodology for analyzing a large number of noise sources in terms of sound pressure and frequency in nine octave bands [1].

\section{Problem}

The main component of broadband noise from a combine harvester's thresher drum is the aero-acoustic noise caused by the processes of formation and disruption of vortices upon the impenetrable surface as well as the turbulent nature of the flow in the boundary layer's area. The profiled character of the rotor beater plates, the different directions of the channels of the previous and subsequent plates (to ensure centering of the threshed mass)

\footnotetext{
${ }^{*}$ Corresponding author: st_fem@bk.ru
} 
are the main sources of vortex formation. The irregularities of the thresher drum's surface itself, in which the rotor plates are mounted, cause the formation of variable pressure values in the impenetrable medium, which makes up another source of noise.

\section{Method}

Sound is defined as an oscillatory movement of the air due to temporary rarefactions and compressions. The movement of a liquid (gas) can be described by two methods: a Lagrangian description of the movement, where the movement of a selected volume element over time is analyzed in a coordinate system of the volume element itself, and the method of representing pressure, density, velocity, acceleration, etc. fields in a fixed coordinate system (Euler coordinates) using vector and tensor calculus.

The motion of a viscous Newtonian fluid is described by a system of partial differential equations - Navier-Stokes equations [2].

$$
\begin{gathered}
\frac{\partial u}{\partial x}+\frac{\partial v}{\partial y}+\frac{\partial w}{\partial z}=0 \\
\frac{\partial u}{\partial t}+u \frac{\partial u}{\partial x}+v \frac{\partial u}{\partial y}+w \frac{\partial u}{\partial z}=\frac{1}{\rho} \cdot \frac{\partial \rho}{\partial x}+\eta \cdot \Delta u+g_{x} \\
\frac{\partial v}{\partial t}+u \frac{\partial v}{\partial x}+v \frac{\partial v}{\partial y}+w \frac{\partial v}{\partial z}=\frac{1}{\rho} \cdot \frac{\partial \rho}{\partial x}+\eta \cdot \Delta v+g_{y} \\
\frac{\partial w}{\partial t}+u \frac{\partial w}{\partial x}+v \frac{\partial w}{\partial y}+w \frac{\partial w}{\partial z}=\frac{1}{\rho} \cdot \frac{\partial \rho}{\partial x}+\eta \cdot \Delta w+g_{z}
\end{gathered}
$$

where $\frac{\partial v_{i}}{\partial t}$ - the equation of momentum; $\frac{\partial \rho}{\partial x_{i}}$ - the density of the surface pressure forces; $\eta \cdot \Delta v_{i}$ - the density of viscous forces; $g_{i}$ - density of mass forces; $\rho$ - density.

The Navier-Stokes equations describe all possible models of fluid and gas flows, however, the limited computational resources make it difficult to obtain acceptable practical cost-effective results, in particular for large Reynolds numbers. To overcome these difficulties, several types of turbulence modeling have been developed: using the Reynolds Averaged Navier-Stokes (RANS) equations; direct numerical simulation of turbulence (Direct Numerical Simulation - DNS); modeling of large eddies (Large Eddy Simulation LES); joint use of Reynolds Averaged Navier-Stokes equations and large eddy modeling (RANS and LES) [3-8]

\section{Decision}

In this paper, the acoustic analysis (the analysis of the broadband noise of a rotating thresher drum) was carried out in the following order:

1. The stationary analysis to determine aero-acoustic sound sources and to establish the cutoff frequency reproduced by the mesh model. Based on the results of stationary analysis, we obtain preliminary data for the subsequent transient analysis: Curle Surface Acouctic Power $\mathrm{dB}$ Contour, that is, the noise level of the turbulent boundary layer of the model surfaces; Proudman Acoustic Power Conrour fields; Mesh Frequency Cutoff Contour. Based on these data, the mesh size is refined and the probes are created in the area of the alleged noise sources.

2. The transient (implicit scheme) analysis. Based on the results of transient analysis, we obtain the following data: dependence of the maximum pressure on time, the values of average flow velocities, the spectral analysis of the broadband noise, the graphs of the 
Proudman acoustic volume power at the control points.

All the existing variety of drum-type threshing machines for combine harvesters can be generalized in terms of their drum diameter $\mathrm{D}=600-660 \mathrm{~mm}$ (John Deer, Deutz-Fahr) and $\mathrm{D}=750-800 \mathrm{~mm}$ (New Holland, PCM), drum rotation frequency 300-1250 rpm and their drum length $\mathrm{L}=1200-1600 \mathrm{~mm}$ [9-15].

A drum with the diameter of $800 \mathrm{~mm}$ was taken as the model under study; the calculations were carried out at the rotation speed of $1050 \mathrm{rpm}$. A general structure of the thresher drum is shown in Figure 1.

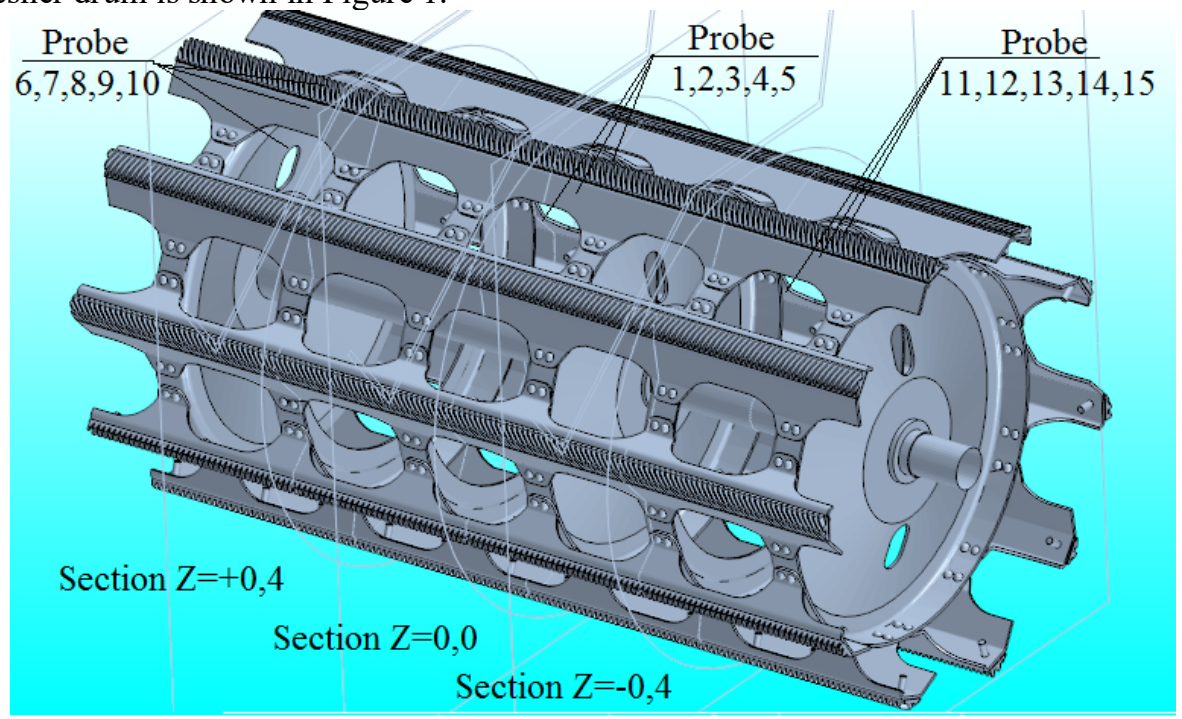

Fig. 1. Thresher drum harvester

Upon the completion of the stationary analysis stage, as the result, the mesh model was refined. The final parameters of the mesh model are following: the total number of mesh cells is 17003634, the size of the surface mesh on the beater plates is $2 \mathrm{~mm}$ and the number of layers of an ordered volume mesh on the drum surface is 4 . There were selected five transverse and two longitudinal control sections and twenty two points (probes) with the purpose of monitoring local changes in pressure and acoustic power. The calculation was performed in steps of $2 \times 10^{-4} \mathrm{~s}$, the total number of iterations was 1415 , and the physical time was $0.1 \mathrm{~s}$.

\section{Results}

Figures 2 and 3 show the scalar and vector fields of average flow rates. Figure 4 shows the acoustic power fields for the drum elements. Figure 5 shows the spectrum of broadband noise. 


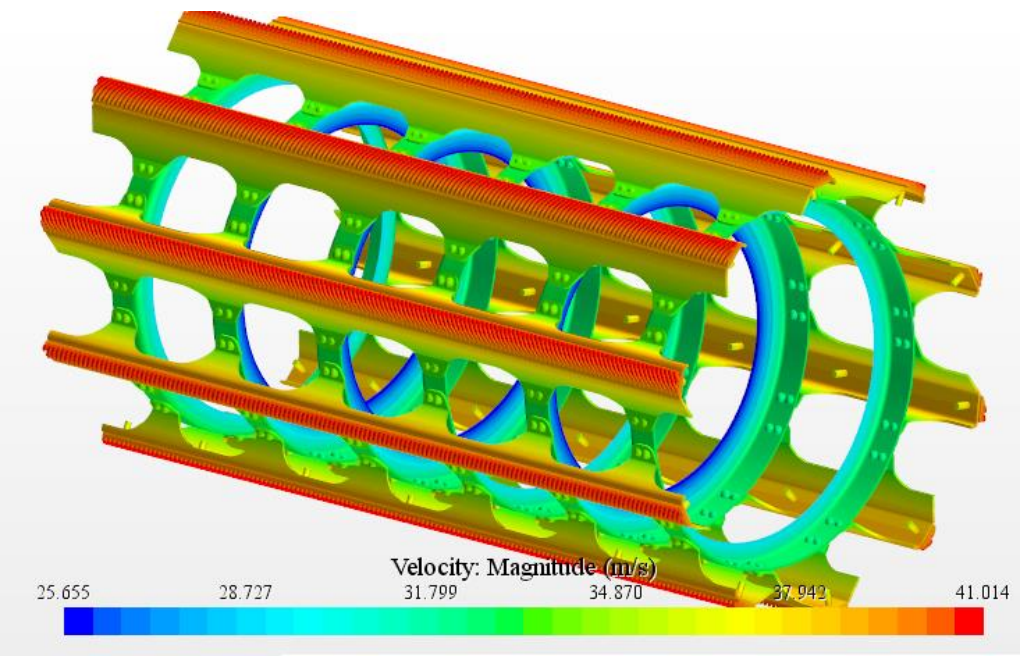

Fig. 2. Contour scalar velocity magnitude

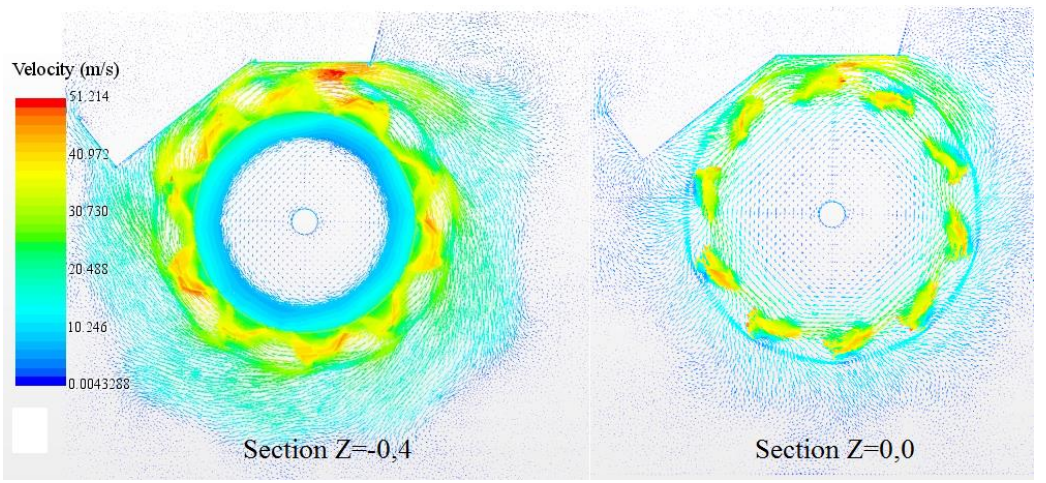

Fig. 3. Contour vector velocity magnitude

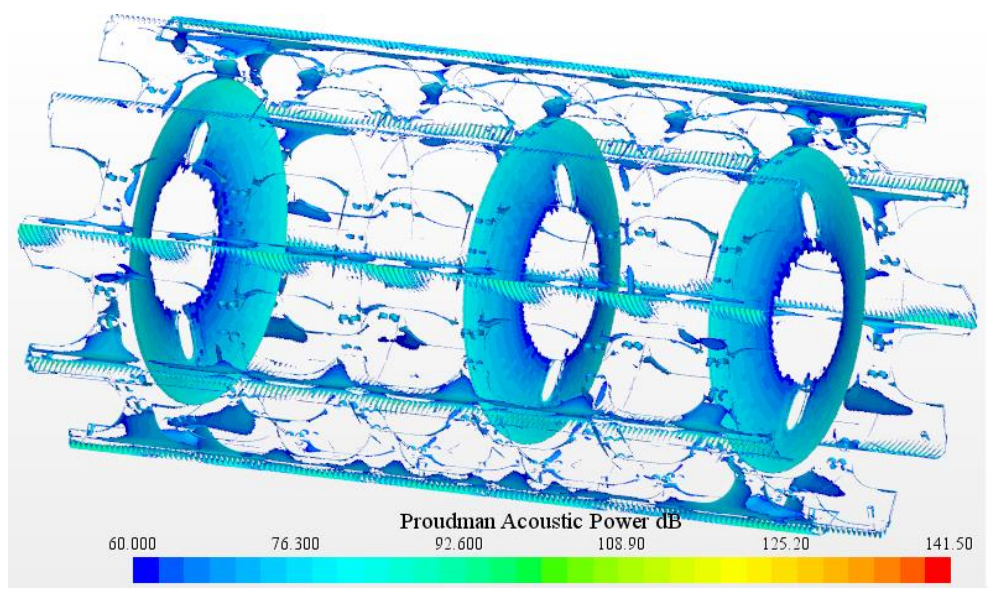

Fig. 4. Proudman acoustic power fields 


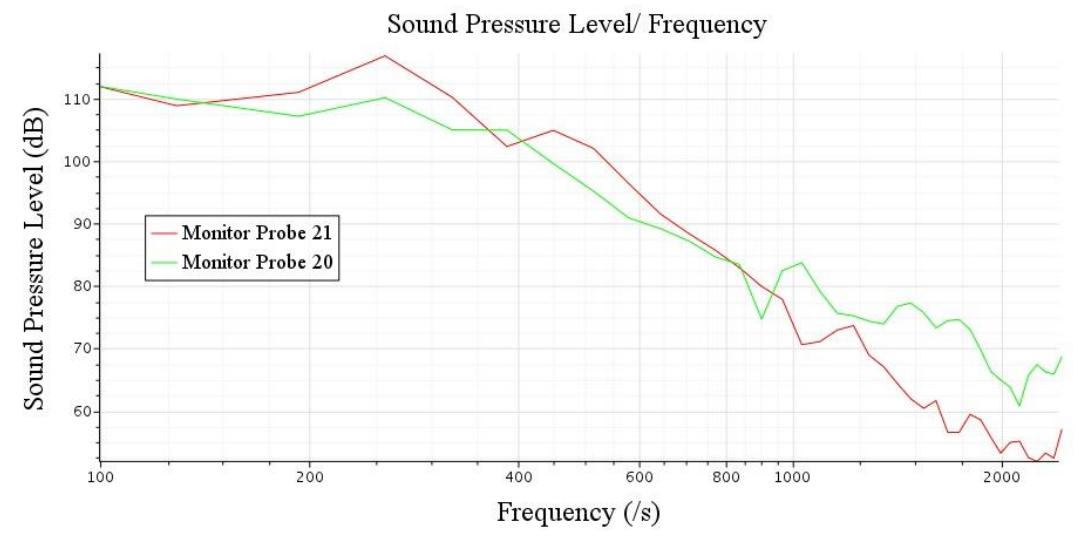

Fig. 5. Contour broad-noise spectrum

The graphs of Figures 6 to 8 show the curves of changes in acoustic power at control points (probes) over time.

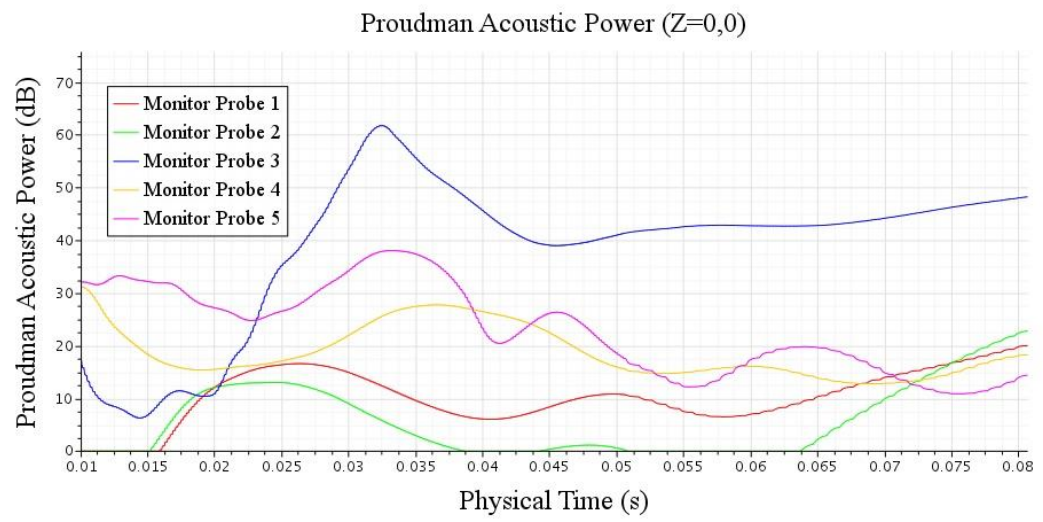

Fig. 6. Variation Proudman acoustic power at control points Probes 1 to 5 over time, section $Z=0,0$

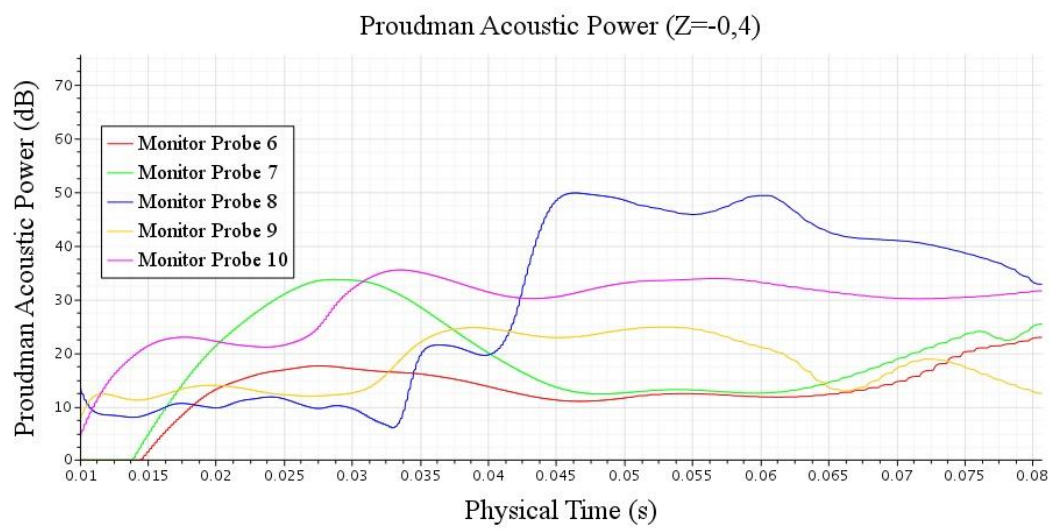

Fig. 7. Variation Proudman acoustic power at control points Probes 6 to 10 over time, section $Z=-0,4$ 


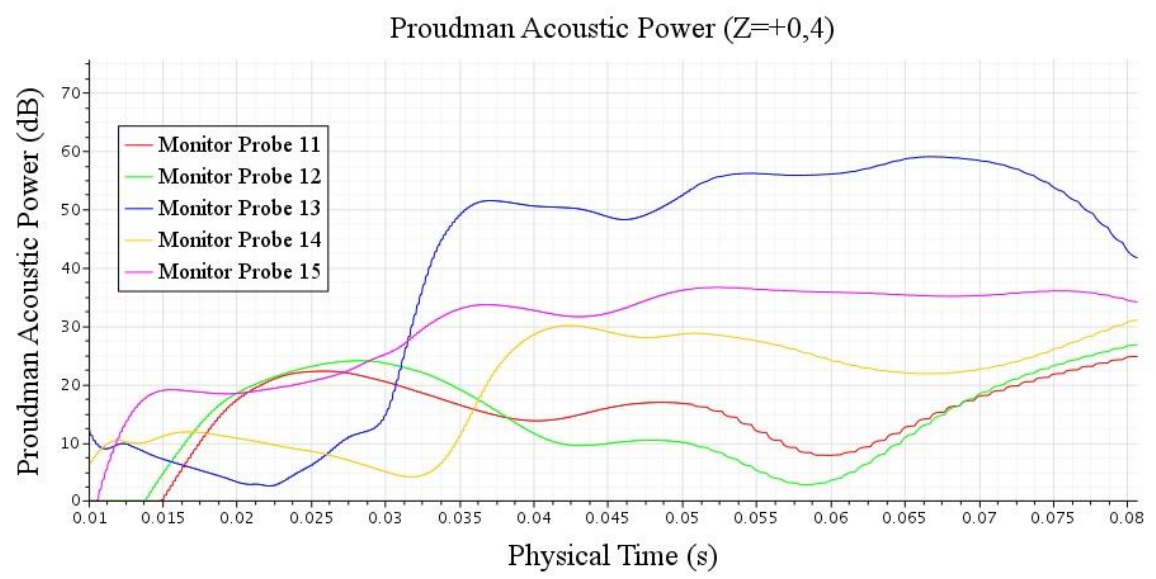

Fig. 8. Variation Proudman acoustic power at control points Probes 11 to 15 over time, section $\mathrm{Z}=+0,4$

\section{Discussion}

The analysis of the results in Figures 5 to 8 allows us to draw the following conclusions:

1 - the maximum sound pressure level is $117 \mathrm{~dB}$;

2 - the maximum sound pressure range falls between the frequencies of $200-300 \mathrm{~Hz}$;

3 - the source of broadband noise must be the turbulent bluff flow in the channels of the beater plates and on the rivets mounting the plates.

\section{Conclusions}

Based on the conclusions drawn, we would like to propose the following direction of further research on this topic: in further calculations it is necessary to revise the position of control points (probes), analyze one module of a rotor beater plate to clarify the nature of the turbulent bluff flow and take into account the acoustic effect of the walls as metal reflectors.

\section{References}

1. J. Struff. The Theory of Sound. (Cambridge University Press, 2011) DOI: 10.1017/CBO9781139058087

2. M. Bruneau. Fundamentals of Acoustics 511-575 (John Wiley \& Sons, Ltd, 2010) DOI: $10.1002 / 9780470612439 . c h 9$

3. C. Laurent, I. Mar, V. Gleize, A. Lerat, D. Arnal. Computers \& Fluids, 61, 21-30 (2012) DOI: 10.1016/j.compfluid.2011.07.011

4. N. Papaxanthos, E. Perrey-Debain, S. Bennouna, B. Ouedraogo, S. Moreau, J.M. Ville. Journal of Sound and Vibration, 393, 176-186 (2017) DOI: 10.1016/j.jsv.2017.01.030

5. T. Colonius, S. Lele. Progress in Aerospace Sciences, 40, 345-416 (2004) DOI: 10.1016/j.paerosci.2004.09.001 
6. S. Deck, P. Weiss, N. Renard. Journal of Computational Physics, 363, 231-255 (2018) DOI: $10.1016 /$ j.jcp.2018.02.028

7. H. Gopalan, S. Heinz, M. Stöllinger. Journal of Computational Physics, 249, 249-274 (2013) DOI: 10.1016/j.jcp.2013.03.066

8. G. Yoon. Computer Methods in Applied Mechanics and Engineering, 361, 112784 (2020) DOI: 10.1016/j.cma.2019.112784

9. Z. Lianga, L. Xu, J. De Baerdemaeker, Y. Li, W. Saeysb. Biosystems Engineering, 190, 25-40 (2020) DOI: 10.1016/j.biosystemseng.2019.11.016

10. S. Sümer, S. Say, F. Ege, A. Sabanci. Applied Ergonomics, 37, 749-756 (2006) DOI: 10.1016/j.apergo.2005.11.006

11. S. Vanbeveren, R. Spinelli, M. Eisenbies, J. Schweier, B. Mola-Yudego, N. Magagnotti, M. Acuna, I. Dimitriou, R. Ceulemans. Renewable and Sustainable Energy Reviews, 76, 90-104 (2017) DOI: 10.1016/j.rser.2017.02.059

12. M. Gebrehiwot, J. De Baerdemaeker, M. Baelmans. Biosystems Engineering, 105, 247-256 (2010) DOI: 10.1016/j.biosystemseng.2009.11.003

13. P. Miu, H. Kutzbach. Computers and Electronics in Agriculture, 60, 105-109 (2008) DOI: 10.1016/j.compag.2007.07.004

14. L. Lizhang, Z. Baijun, L. Yaoming. Computers and Electronics in Agriculture, 152, 141-148 (2018) DOI: 10.1016/j.compag.2018.07.019

15. Y. Zhang, D. Chen, Y. Yin, X. Wang, S. Wang. IFAC-PapersOnLine, 51, 402-407 (2018) DOI: 10.1016/j.ifacol.2018.08.188 